\title{
Some Exact Results \\ for the Zero-Bandwidth Extended Hubbard Model with Intersite Charge and Magnetic Interactions
}

\author{
K.J. KapCiA*, W. KŁobus And S. RobaszkiEWICZ \\ Faculty of Physics, Adam Mickiewicz University in Poznań, \\ Umultowska 85, 61-614 Poznań, Poland
}

\begin{abstract}
The extended Hubbard model in the zero-bandwidth limit is studied. The effective Hamiltonian consists of (i) on-site $U$ interaction, (ii) intersite density-density interaction $W$, and (iii) Ising-like magnetic exchange interaction $J$ between the nearest-neighbors. We present rigorous (and analytical) results obtained within the transfer-matrix method for $1 \mathrm{D}$ chain in two particular cases: (a) $W=0$ and $n=1$; (b) $U \rightarrow+\infty$ and $n=1 / 2$ $(W \neq 0, J \neq 0)$. We obtain the exact formulae for the partition functions which enables to calculate thermodynamic properties such as entropy, specific heat $(c)$, and double occupancy per site. In both cases the system exhibits an interesting temperature dependence of $c$ involving a characteristic two-peak structure. There are no phase transitions at finite temperatures and the only transitions occur in the ground state.
\end{abstract}

DOI: $10.12693 /$ APhysPolA.127.284

PACS: 71.10.Fd, 71.10.-w, 71.10.Hf, 71.45.Lr, 75.30.Fv

\section{Introduction}

The interplay between density-density and magnetic interactions is relevant to a broad range of important materials such as manganites, multiferroics, organics, and other strongly correlated electron systems [1-9].

In this paper we present some exact results obtained within transfer matrix method for the zero-bandwidth extended Hubbard model with density-density and Isinglike magnetic interactions on the one-dimensional chain $(d=1)$. The 1D-Hamiltonian considered has a form

$$
\hat{H}=\sum_{i=1}^{L}\left[U \hat{n}_{i \uparrow} \hat{n}_{i \downarrow}+W \hat{n}_{i} \hat{n}_{i+1}-4 J \hat{s}_{i}^{z} \hat{s}_{i+1}^{z}-\mu \hat{n}_{i}\right],
$$

where $\hat{c}_{i \sigma}^{+}$denotes the creation operator of an electron with $\operatorname{spin} \sigma(\sigma=\uparrow, \downarrow)$ at site $i, \hat{n}_{i \sigma}=\hat{c}_{i \sigma}^{+} \hat{c}_{i \sigma}, \hat{n}_{i}=\sum_{\sigma} \hat{n}_{i \sigma}$, and $\hat{s}_{i}^{z}=(1 / 2)\left(\hat{n}_{i \uparrow}-\hat{n}_{i \downarrow}\right) . i+1$ is the nearest neighbor of the $i$-site in the chosen direction (from two possible directions in a chain). We assume the periodic boundary conditions, i.e. $n_{L+1 \sigma}=n_{1 \sigma}$, where $L$ is a number of sites in the chain and $n_{i \sigma}=\left\langle\hat{n}_{i \sigma}\right\rangle . J_{0}=z J$, where $z=2$ is a number of the nearest neighbors.

All the terms of Hamiltonian (1) commute with one another and are diagonal in the representation of occupancy numbers. It is convenient to use the transfer matrix method [10] to find the grand partition function $Z$.

Hamiltonian (1) can be treated as a simple effective model of insulators, in which interactions $U, W$ and $J$ are assumed to include all the possible contributions and renormalizations. Notice that ferromagnetic $(J>0)$ interactions are simply mapped onto the antiferromagnetic ones $(J<0)$ by redefining the spin direction on one sublattice in lattices decomposed into two interpenetrating

*corresponding author; e-mail: konrad.kapcia@amu.edu.pl

sublattices. Thus, we restrict ourselves to a case of $J>0$.

Exact solutions of model (1) for some particular cases have been obtained for the one-dimensional case $(T \geq 0)$ employing the method based on the equations of motion and Green function formalism [11-13] or the transfermatrix method [14-17]. Extensive mean-field studies (exact result in $d \rightarrow+\infty$ ) [18-27] and some Monte Carlo simulations $(d=2)$ [28-29] of model (1) have been also performed. Moreover, the exact ground state $(T=0)$ results have been found for $2 \leq d<+\infty[30-34]$.

We present rigorous results for partition functions obtained within the transfer-matrix method for onedimensional model (1) in two particular cases: (a) $W=0$ and $n=1$; (b) $U \rightarrow+\infty$ and $n=1 / 2(W \neq 0, J \neq 0)$.

\section{Results and discussion}

(a) For the case of $W=0$, a typical element of the transfer matrix for model (1) is defined as

$$
\begin{aligned}
& P_{i, i+1} \equiv\left\langle n_{i \uparrow} n_{i \downarrow}|P| n_{i+1 \uparrow} n_{i+1 \downarrow}\right\rangle= \\
& \exp \left(-\beta\left[(U / 2)\left(n_{i \uparrow} n_{i \downarrow}+n_{i+1 \uparrow} n_{i+1 \downarrow}\right)-(\mu / 2) n_{i}\right.\right. \\
& \left.\left.-(\mu / 2) n_{i+1}-J\left(n_{i \uparrow}-n_{i \downarrow}\right)\left(n_{i+1 \uparrow}-n_{i+1 \downarrow}\right)\right]\right),
\end{aligned}
$$

where $\left|n_{i \uparrow} n_{i \downarrow}\right\rangle \in\{|00\rangle,|01\rangle,|10\rangle,|11\rangle\}$ denotes a singlesite state at site $i, \beta=1 /\left(k_{\mathrm{B}} T\right)$ is the inverse temperature and $k_{\mathrm{B}}$ is the Boltzmann constant. One obtains 16 matrix elements and the problem is reduced to diagonalization of the matrix $\check{P}$ of the form

$$
\check{P}=\left(\begin{array}{cccc}
1 & x_{0} & x_{0} & u_{0} x_{0}^{2} \\
x_{0} & m_{0} x_{0}^{2} & m_{0}^{-1} x_{0}^{2} & u_{0} x_{0}^{3} \\
x_{0} & m_{0}^{-1} x_{0}^{2} & m_{0} x_{0}^{2} & u_{0} x_{0}^{3} \\
u_{0} x_{0}^{2} & u_{0} x_{0}^{3} & u_{0} x_{0}^{3} & u_{0}^{2} x_{0}^{4}
\end{array}\right),
$$

where $x_{0}=\exp (\beta \mu / 2), u_{0}=\exp (-\beta U / 2)$ and $m_{0}=$ $\exp \left(\beta J_{0} / 2\right)$. One can show that three eigenvalues of $\check{P}$ $\left(\lambda_{l}, l=1,2,3\right)$ are roots of a cubic equation

$$
\lambda^{3}-\lambda^{2}\left(1+2 m_{0} x+u x^{2}\right)-\lambda\left\{x m_{0}^{-2}\left(1-m_{0}\right)\right.
$$




$$
\begin{aligned}
& \left.\times\left[x+m_{0} x+m_{0}^{3} x+m_{0}^{2}\left(2+x+2 u x^{2}\right)\right]\right\} \\
& +x^{2} m_{0}^{-2}\left(1-m_{0}\right)^{3}\left(1+m_{0}\right)\left(1+u x^{2}\right)=0,
\end{aligned}
$$

where $x=x_{0}^{2}$ and $u=u_{0}^{2}$, while $\lambda_{4}=0$.

So far the number $N$ of particles in the chain has not been specified. It can be done in a standard way by solving the following equation: $N=-(\partial \Omega / \partial \mu)_{\beta}$, where $\Omega$ is the grand canonical potential, $\Omega=-k_{\mathrm{B}} T \ln Z$. In the thermodynamic limit $L \rightarrow \infty$ the grand sum of states $Z$ is derived as $Z=\lambda_{\mathrm{M}}^{L}$, where $\lambda_{\mathrm{M}}$ is the maximum eigenvalue of $\check{P}$ (assumed to be nondegenerate). Therefore, the equation for $N$ can be rewritten as $\partial \lambda_{\mathrm{M}} / \partial x=n \lambda_{\mathrm{M}} / x$, where $n=N / L$ is electron density in a system.

In the case of half-filling $(n=1)$, the condition for $N$ can be solved analytically for arbitrary $U$ and in such a case the chemical potential is derived as $\mu=U / 2$ and $\lambda_{\mathrm{M}}$ takes the form

$$
\lambda_{\mathrm{M}}=1+\exp \left(\frac{\beta U}{2}\right) \cosh \left(\frac{\beta J_{0}}{2}\right)+\frac{X}{2} \exp \left(\frac{\beta Y}{2}\right),
$$

where $X=\sqrt{1+Z_{1}-4 Z_{2}-4 Z_{3}+16 Z_{4}+4 Z_{5}+2 Z_{6}}$, $Y=U-J_{0}, Z_{1}=\exp \left(2 \beta J_{0}\right), Z_{2}=\exp (-\beta Y / 2)$, $Z_{3}=\exp (\beta A / 2), Z_{4}=\exp (\beta B / 2), Z_{5}=\exp (-\beta Y)$, $Z_{6}=\exp \left(\beta J_{0}\right)$, and $A=3 J_{0}-U, B=2 J_{0}-U$.

(b) The limit $U \rightarrow+\infty$ corresponds to the subspace where the double occupancy of sites is excluded (by electrons for $n<1$ or holes for $n>1$ ). For this case the transfer matrix elements for model (1) are defined as

$$
\begin{aligned}
& R_{i, i+1} \equiv\left\langle n_{i \uparrow} n_{i \downarrow}|R| n_{i+1 \uparrow} n_{i+1 \downarrow}\right\rangle= \\
& \quad \exp \left(-\beta\left(W\left(n_{i \uparrow}+n_{i \downarrow}\right)\left(n_{i+1 \uparrow}+n_{i+1 \downarrow}\right)\right.\right. \\
& \left.\left.\quad-(\mu / 2)\left(n_{i}+n_{i+1}\right)-J\left(n_{i \uparrow}-n_{i \downarrow}\right)\left(n_{i+1 \uparrow}-n_{i+1 \downarrow}\right)\right)\right)
\end{aligned}
$$

where $\left|n_{i \uparrow} n_{i \downarrow}\right\rangle \in\{|00\rangle,|01\rangle,|10\rangle\}$ denotes a single-site state at site $i$ in the limit $U \rightarrow+\infty$. Therefore, in this case the matrix $\check{R}$ has the following form:

$$
\check{R}=\left(\begin{array}{ccc}
1 & x_{0} & x_{0} \\
x_{0} & m_{0} x_{0}^{2} w_{0} & m_{0}^{-1} x_{0}^{2} w_{0} \\
x_{0} & m_{0}^{-1} x_{0}^{2} w_{0} & m_{0} x_{0}^{2} w_{0}
\end{array}\right),
$$

where $x_{0}=\exp (\beta \mu / 2), w_{0}=\exp \left(-\beta W_{0} / 2\right)$ and $m_{0}=$ $\exp \left(\beta J_{0} / 2\right)$. The eigenvalues of $\check{P}$ are roots $\lambda_{l}(l=$ $1,2,3)$ of the following cubic equation:

$$
\lambda^{3}-\lambda^{2}\left(1+2 m_{0} x w_{0}\right)
$$

$$
\begin{aligned}
& -\lambda x\left[2-2 m_{0} w_{0}+x w\left(m_{0}^{-2}-m_{0}^{2}\right)\right] \\
& +\left(1-m_{0}^{-2}\right) x^{2} w\left(1+m_{0}^{2}-2 m_{0} w_{0}^{-1}\right)=0,
\end{aligned}
$$

where $x=x_{0}^{2}$ and $w=w_{0}^{2}$.

In this case the equation for $N$ can be solved analytically for $n=1 / 2$. One finds that $\mu=W_{0} / 2-k_{\mathrm{B}} T \ln \left(2 \cosh \left(\beta J_{0} / 2\right)\right)$, and $\lambda_{\mathrm{M}}$ is derived

$$
\lambda_{\mathrm{M}}=1+\exp \left(\beta W_{0} / 4\right) \sqrt{\operatorname{sech}\left(\beta J_{0} / 2\right)} .
$$

The knowledge of explicit form of the sum of states $Z$ allows us to obtain thermodynamic characteristics of the system for arbitrary temperature. Local magnetic moment $\gamma$ is defined by: $\gamma=(1 / 2 L) \sum_{i}\left\langle\left|n_{i \uparrow}-n_{i \downarrow}\right|\right\rangle$. It is related with the double occupancy $D$ per site (defined by the formula: $\left.D=(1 / L)\left\langle\hat{n}_{i \uparrow} \hat{n}_{i \downarrow}\right\rangle=(\partial f / \partial U)_{T}\right)$ by the relation: $\gamma=n / 2-D$, where $f=\omega+n \mu$ is the free energy of the system per site $(\omega \equiv \Omega / L)$. The entropy $s$ and the specific heat $c$ (per site) can be derived as: $s=-\partial f / \partial T$ and $c=-T\left(\partial^{2} f / \partial T^{2}\right)$. Because the explicit forms of the partition function $Z$ in both cases are known and the derivation of the above thermodynamical characteristics (i.e. $D, s, c$ ) is rather straightforward $\left(\omega=-k_{\mathrm{B}} T \ln \lambda_{\mathrm{M}}\right)$, below we only summarize the most important conclusions following from the analysis of Eqs. (5) and (9).

One can observe that the system exhibits an interesting temperature dependence of $c$ involving a characteristic twopeak structure for some values of model parameters in the cases analyzed. In both cases considered above there are no phase transitions at finite temperatures (in the agreement with Mermin-Wagner theorem [35]) and the only transitions can occur in the ground state.

$$
\text { 2.1. (a) The case of } W=0 \text { and } n=1
$$

For large $U / J_{0} c$ exhibit two-peak structure, whereas for $U \lesssim 6$ peaks merge and there is a single peak in $T$-dependence of $c$ (labeled as $T_{1}+T_{2}$, cf. Fig. 1 ). For $-1<U / J_{0} \lesssim-0.9$ two peaks of $c$ appear again. The broad one (at higher temperature $T_{1}$ ) is connected with continuous changes in short-range charge on-site ordering (associated with $U$ term). The narrow one (at lower temperature $T_{2}<T_{1}$ ) is connected with short-range intersite magnetic ordering ( $J$ term). With decrease of $U / J_{0}$ their locations move towards lower temperatures. If $U / J_{0}<-1$ the single maximum of $c$ (connected with short-range on-site ordering) exists only and it moves toward higher temperatures with increasing of $|U| / J_{0}$. In the limit $U \rightarrow+\infty(n=1)$ the specific heat exhibits a single peak described by $c^{I s}=k_{\mathrm{B}}\left[\left(\beta J_{0} / 2\right) \times \operatorname{sech}\left(\beta J_{0} / 2\right)\right]^{2}$, which corresponds to $1 \mathrm{D}$-Ising model in the absence of magnetic field (the peak connected with on-site ordering is "located" at $\left.T_{1} \rightarrow+\infty\right)$. The divergence of $c$ at $U / J_{0} \rightarrow-1$ and $T_{2} \rightarrow 0$ indicates that the first-order transition occurs between the nonordered state of double occupied sites $(\gamma=0)$ and the ferromagnetic homogeneous phase (stable for $U / J_{0}>-1$ ), where all sites are singly occupied $(\gamma=1 / 2)$. One can derive the same conclusion of $T=0$ properties of the system from a behavior of the entropy $s$. For $U / J_{0}>-1$ the system is magnetically ordered with $\bar{s}(0)=0$ (a number of states is $\left.g=2, s=k_{\mathrm{B}} \bar{s}=\left(k_{\mathrm{B}} / L\right) \ln g\right)$. For $U / J_{0}<-1$ the system consists of nonordered on-site electron pairs and $\bar{s}(0)=\ln 2$ $\left(g=2^{L}\right)$. At $U / J_{0} \rightarrow-1 \bar{s}(0)=\ln 2$. Notice that in the limit $T \rightarrow \infty$ the entropy $\bar{s} \rightarrow 2 \ln 2$ for any $U / J_{0}$.

Our results for $W=0$ and $n=1$ are in an agreement with the results of Ref. [11] obtained using the Green function formalism, whereas the numerical analyses of (4) and the condition for $N$ (for arbitrary $n$ or $\mu$ ) should be consistent with the results of Ref. [13].

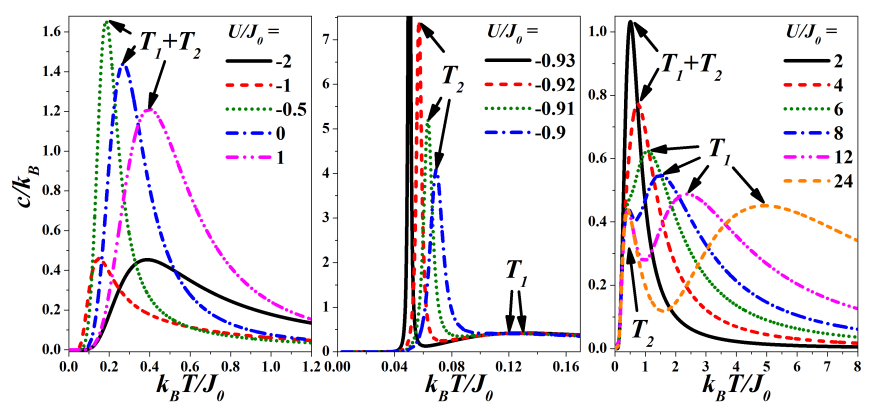

Fig. 1. The specific heat $c$ as a function of $k_{\mathrm{B}} T / J_{0}$ for several values of $U / J_{0}$ (as labeled); $n=1, W=0$. 


\section{2. (b) The case of $n=1 / 2$ for $U \rightarrow+\infty$}

At $T=0$ for $W / J=1$ the transition between the homogeneous charge-ordered $(\mathrm{CO})$ phase (for $W / J>1$ ) and phase separated (PS) state occurs, cf. also Ref. [24]. For $W / J<1$ the system is divided into two equal-sized domains: one ferromagnetically ordered completely filled by electrons $(n=1)$ and the other empty $(n=0)$. The behavior of $c$ at $T>0$ is very similar to that discussed in the previous case ( simplifying, for qualitative discussion only $U / J_{0} \leftrightarrow-W / J$ replacement is needed, short-range charge order peak in $c$ is associated with $W$ term). In the limit $W / J \rightarrow-\infty c$ exhibits a single maximum described by the characteristic dependence for 1D-Ising model, but in such a case the specific heat $c^{*}$ of the system is twice smaller than the result $c^{I s}$ obtained in a case of $n=1, W=0\left(c^{*}=c^{I s} / 2\right)$. It can be derived that at $T=0$ : (i) for $W / J>1: \bar{s}(0)=(1 / 2) \ln 2$ (the CO phase, $g=2^{(L / 2+1)}$ ) and (ii) for $W / J<1: \bar{s}(0)=0$ (the PS:F $/ \mathrm{NO}$ state, $g=2 L)$. If $W=J$ the ground state is highly degenerated and $\bar{s}(0)=\ln (1+\sqrt{2})$ (cf. Fig. 2). In the limit $T \rightarrow \infty$ the entropy $\bar{s} \rightarrow(3 / 2) \ln 2$.
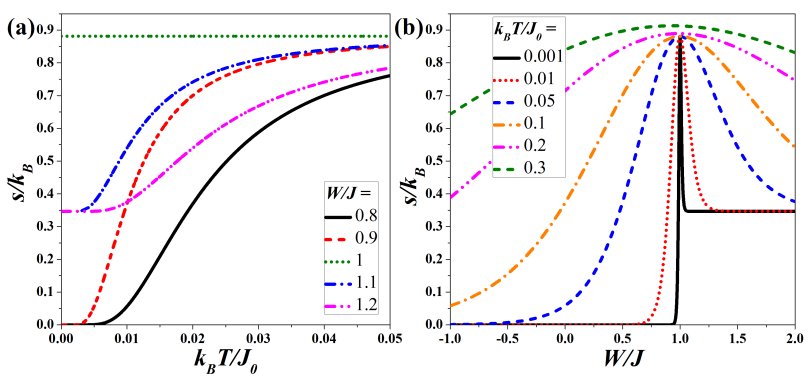

Fig. 2. The entropy $s / k_{\mathrm{B}} \equiv \bar{s}$ as a function (a) of $k_{\mathrm{B}} T / J_{0}$ and (b) of $W / J(U \rightarrow+\infty, n=1 / 2$, values of other model parameters as labeled).

The detailed discussion of thermodynamic properties of one-dimensional model (1) in a general case will be the subject of a subsequent paper.

\section{Acknowledgments}

The work (K.J.K., S.R.) has been financed by National Science Centre (NCN, Poland) as a research project under grant No. DEC-2011/01/N/ST3/00413 and a doctoral scholarship No. DEC-2013/08/T/ST3/00012. K.J.K. thanks for the financial support from the ESF - OP "Human Capital" - POKL.04.01.01-00-133/09-00 — "Proinnowacyjne kształcenie, kompetentna kadra, absolwenci przyszłości". K.J.K. and W.K. thank the Foundation of Adam Mickiewicz University in Poznan for the support from its scholarship programme.

\section{References}

[1] R. Micnas, J. Ranninger, S. Robaszkiewicz, Rev. Mod. Phys. 62, 113 (1990).

[2] T. Goto, B. Lüthi, Adv. Phys. 52, 67 (2003).

[3] E. Dagotto, T. Hotta, A. Moreo, Phys. Rep. 344, 1 (2001).

[4] J. van den Brink, D.I. Khomskii, J. Phys. Condens. Matter 20, 434217 (2008).

[5] W.R. Czart, S. Robaszkiewicz, Acta Phys. Pol. A 106, 709 (2004); W.R. Czart, S. Robaszkiewicz, Phys. Status Solidi B 243, 151 (2006).

[6] W.R. Czart, S. Robaszkiewicz, Acta Phys. Pol. A 109, 577 (2006); W.R. Czart, S. Robaszkiewicz, Mater. Sci.-Poland 25, 485 (2007).
[7] K. Kapcia, Acta Phys. Pol. A 121, 733 (2012).

[8] K. Kapcia, J. Supercond. Nov. Magn. 26, 2647 (2013).

[9] K.J. Kapcia, Acta Phys. Pol. A 127, 204 (2015); K.J. Kapcia, J. Supercond. Novel Magn. in press (2015); DOI:10.1007/s10948-014-2906-4 .

[10] G.F. Newell, E.W. Montroll, Rev. Mod. Phys. 25, 353 (1953).

[11] F. Mancini, F.P. Mancini, Phys. Rev. E 77, 061120 (2008).

[12] F. Mancini, E. Plekhanov, G. Sica, Cent. Eur. J. Phys. 10, 609 (2012).

[13] F. Mancini, E. Plekhanov, G. Sica, Eur. Phys. J. B 86, 224 (2013).

[14] F. Mancini, E. Plekhanov, G. Sica, J. Phys. Conf. Series 391, 012148 (2012).

[15] R.A. Bari, Phys. Rev. B 3, 2662 (1971).

[16] G. Beni, P. Pincus, Phys. Rev. B 9, 2963 (1974).

[17] R.S. Tu, T.A. Kaplan, Phys. Status Solidi B 63, 659 (1974).

[18] R. Micnas, S. Robaszkiewicz, K.A. Chao, Phys Rev. B 29, 2784 (1984).

[19] K. Kapcia, W. Kłobus, S. Robaszkiewicz, Acta. Phys. Pol. A 118, 350 (2010).

[20] K. Kapcia, S. Robaszkiewicz, J. Phys. Condens. Matter 23, 105601 (2011).

[21] K. Kapcia, S. Robaszkiewicz, J. Phys. Condens. Matter 23, 249802 (2011).

[22] K. Kapcia, S. Robaszkiewicz, Acta Phys. Pol. A 121, 1029 (2012)

[23] W. Kłobus, K. Kapcia, S. Robaszkiewicz, Acta. Phys. Pol. A 118, 353 (2010).

[24] K. Kapcia, W. Kłobus, S. Robaszkiewicz, Acta Phys. Pol. A 121, 1032 (2012).

[25] S. Robaszkiewicz, Phys. Status Solidi B 59, K63 (1973).

[26] S. Robaszkiewicz, Phys. Status Solidi B 70, K51 (1975).

[27] S. Robaszkiewicz, Acta Phys. Pol. A 55, 453 (1979).

[28] S. Murawski, K. Kapcia, G. Pawłowski, S. Robaszkiewicz, Acta Phys. Pol. A 121, 1035 (2012).

[29] S. Murawski, K.J. Kapcia, G. Pawłowski, S. Robaszkiewicz, Acta Phys. Pol. A 127, 281 (2015).

[30] S. Murawski, K. Kapcia, G. Pawłowski, S. Robaszkiewicz, Acta Phys. Pol. A 126, A110 (2014).

[31] S.A. Pirogov, Ya.G. Sinai, Theor. Math. Phys. 25 1185 (1975); S.A. Pirogov, Ya.G. Sinai, Theor. Math Phys. 26, 39 (1976).

[32] J. Jędrzejewski, Z. Phys. B 48, 219 (1982); J. Jędrzejewski, Z. Phys. B 59, 325 (1985).

[33] U. Brandt, J. Stolze, Z. Phys. B 62, 433 (1986).

[34] J. Jędrzejewski, Physica A 205, 702 (1994).

[35] N.D. Mermin, H. Wagner, Phys. Rev. Lett. 17, 1133 (1966). 